See discussions, stats, and author profiles for this publication at: https://www.researchgate.net/publication/334323645

\title{
Multiobjective optimization of modular structures: Weight versus geometric versatility in a Truss-Z system
}

Article in Computer-Aided Civil and Infrastructure Engineering · July 2019

DOI: $10.1111 /$ mice. 12478

CITATIONS

0

2 authors:

Machi Zawidzki

Institute of Fundamental Technological Research

61 PUBLICATIONS 165 CITATIONS

SEE PROFILE
40

Łukasz Jankowski

Institute of Fundamental Technological Research

79 PUBLICATIONS 379 CITATIONS

SEE PROFILE

Some of the authors of this publication are also working on these related projects:

Innovative Extremely Modular Systems for temporary and permanent deployable structures and habitats: development, modeling, evaluation \& optimization View project 
See discussions, stats, and author profiles for this publication at: https://www.researchgate.net/publication/334323645

\section{Multiobjective optimization of modular structures: Weight versus geometric versatility in a Truss $-Z$ system}

Article in Computer-Aided Civil and Infrastructure Engineering • July 2019

DOI: $10.1111 /$ mice.12478

CITATIONS

0

2 authors:

Machi Zawidzki

Institute of Fundamental Technological Research

58 PUBLICATIONS 153 CITATIONS

SEE PROFILE
READS

29

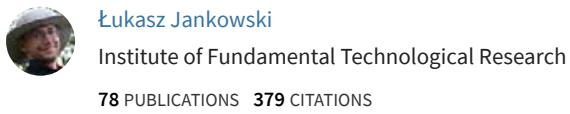

78 PUBLICATIONS 379 CITATIONS

SEE PROFILE

Some of the authors of this publication are also working on these related projects:

Innovative Extremely Modular Systems for temporary and permanent deployable structures and habitats: development, modeling, evaluation \& optimization View project 
This is the peer reviewed version of the following article:

Zawidzki M, Jankowski $Ł$. Multiobjective optimization of modular structures:

\title{
Multiobjective optimization of modular structures: weight versus geometric versatility in a Truss- $Z$ system
}

\author{
Machi Zawidzki I Łukasz Jankowski
}

Institute of Fundamental Technological Research, Polish Academy of Sciences, Poland

\section{*Correspondence}

Machi Zawidzki, Institute of Fundamental Technological Research, Polish Academy of Sciences, Warsaw, Poland.

Email: zawidzki@mit.edu

\begin{abstract}
This paper proposes an approach for multicriterial optimization of modular structures with respect to their structural and geometrical properties. The approach is tested using the quickly deployable and reconfigurable modular ramp system Truss$\mathrm{Z}$ intended for pedestrian traffic. The focus is on modular structures composed of a moderate number of relatively complex modules, which feature an irregular, noncuboidal geometry. Such modules can be assembled into a variety of geometrically different configurations which do not adhere to any predefined spatial grid; their global geometry can be treated as free-form and determined in-situ during construction. The optimization variables represent local-level geometrical and structural properties of a single module. The Pareto front is used to balance between two kinds of objectives. The geometrical objective quantifies the ability of the modules to generate geometrically versatile global structures that are well-suited to comply with spatial constraints of real construction sites. The structural objective is formalized in analogy to the minimum weight problem with upper bound constraints imposed on the von Mises stress and the Euler buckling load ratio. A two level optimization scheme is employed with NSGA-II at the top level and a simulated annealing with adaptive neighborhood at the lower level.
\end{abstract}

\section{1 | INTRODUCTION}

Mass prefabrication and modularity are the common ways to minimize the cost and time of construction (Smith, 2011). However, they substantially limit the diversity of attainable forms, so that the resulting structures are suboptimal. This is the price for the economization, which should be alleviated by optimizing such structures within the constraints imposed by their modularity. The field of structural optimization has already been flourishing for decades, see, e.g., Deaton \& Grandhi (2013); Sigmund \& Maute (2013) for reviews, Plevris \& Papadrakakis (2010), or Adeli \& Kamal (19861991) for early research on optimization of trusses. Astonishingly, it seems that optimization of modular structures still constitutes a relatively unexplored area with only a limited number of published results.

Tugilimana, Thrall, \& Coelho (2017) consider girder-type bridges comprised of stacked rectangular truss panels. Subject to optimization is the internal topology of the panels and their in-place spatial orientation. To ensure the geometric compatibility, the allowable orientations of a panel preserve the coordinates of the interface degrees of freedom (Dofs) and amount effectively to rotations and mirror reflections. In comparison to the earlier work by the same authors (Tugilimana, Thrall, Descamps, \& Coelho, 2017), the proposed formulation circumvents discrete optimization by encoding the spatial orientation with continuous variables, while the geometric compatibility of the converged solution is enforced by additional constraints. Torstenfelt \& Klarbring (2006) consider 
families of related products, where certain modules are shared between specific products. A proof-of-concept framework is proposed for product optimization and illustrated in a case study of a car frame family that is comprised of the base model and its seven-seat and pickup versions, which all share two frame fragments.

A related research stream deals with topology optimization of periodic structures, see for example C. W. Zhou, Lainé, Ichchou, \& Zine (2015) or Moses, Fuchs, \& Ryvkin (2002). The common trait is the congruency of modules or unit cells. However, the units in periodic structures must be arranged on a predesigned and very regular spatial grid with exact topology repetition, which is a strong constraint. In contrast, in modular structures:

- the ultimate spatial configuration of any module might be unknown in advance,

- an irregular geometry of the module might lead to a significant geometric diversity of the resulting global structures. In the extreme situation, as considered in this paper, the global geometry may be treated as free-form and determined in-situ during construction.

Certain analogues can be also found in the area of multiscale (and often multiphysical) material modeling with representative volume elements (RVE) used at the microscale level (N. Chen, Yu, Xia, Liu, \& Ma 2017, Y. Chen, Zhou, \& Li, 2010, Wang, Luo, Zhang, \& Qin, 2016) or lattice-structured materials with mesoscale cells (Augustyniak, 2018, Messner, 2016). However, typical optimization objectives express selected properties of the homogenized material itself, and they usually do not involve global-level properties as typical in structural optimization and structural mechanics. A relatively rare exception is the research reported in Liu, Yan, \& Cheng (2008) and Niu, Yan, \& Cheng (2008), where the global structure is optimized concurrently with the cell microstructure using the homogenization approach, and the objectives at the global level are respectively the minimum compliance and the maximum fundamental natural frequency.

This paper focuses on optimization of modular structures, which-in contrast to the structures periodic at the microand mesoscales - are composed of a relatively low number of relatively large and complex modules. In such a case, direct application of the periodicity-based optimization approaches might result in trivial and repetitive global forms. Therefore, a noncuboidal geometry is used for 3D modules, instead of assuming that the unit cells fill a regular rasterization grid for the global structure. The modules have at least two faces congruent, which allows them to be connected to each other in a number of geometrically different configurations. As a result, free-form global structures can be achieved that are not constrained by any regular tessellation of space. As a motivation and an illustrative example of the application context, a rapidly deployable and quickly reconfigurable modular ramp system for pedestrian traffic is used (Zawidzki 2015).

During design and optimization of such a modular structure, two general groups of criteria need to be taken into account:

1. criteria related to the outer geometry of the module, which directly affect the ability of the modules to create a variety of global structures and global geometries. Such criteria need to be quantified at the global level, by considering the global geometrical versatility and geometry-related functional requirements;

2. typical structural optimization criteria, which depend on the assumed loading conditions, the internal topology of the module and sizing of its elements.

In the previous research, the geometry of the module has been assumed in advance and kept constant, so that the optimization has been solely focused either on the global geometry of the assembled structure or on the structural criteria. That is either:

- the subject of optimization has been the shape of the global structure in a given environment, which is ultimately a combinatorial problem of finding a discrete spatial configuration of modules, where the optimality criteria and constraints are purely geometrical in nature, see, e.g., Zawidzki (2015), or

- the total mass of the module and its internal configuration have been optimized in a typical sizing problem, but the geometry of the module has been kept constant (Zawidzki \& Jankowski, 2018).

Here, an approach is presented that uses a two-level, multicriterial optimization to concurrently address both types of criteria (Ehrgott, 2005, Marler \& Arora. 2004), and which is inspired by the interactive layout optimization presented in Adeli \& Balasubramanyam (1987). The geometry of the module is no longer assumed in advance and constant: the optimization variables represent local geometrical and structural properties of a single module. The subject of optimization is the total module mass (subject to stress/force constraints) and the geometrical versatility of the resulting modular system. The latter is understood as the ability to generate geometrically diverse global structures with possibly uniform coverage of the spatial environment at a possibly uniform directionality of the exit module. Such a geometric measure is intended to quantify the suitability of the system to comply with intricate spatial constraints of real construction sites. In comparison to the existing approaches, such an optimization approach is untypical and original: although there is essentially a single module,

- it is used within the global structure in a variety of localizations to perform various structural roles, 
- it is used to construct diverse global structures with very different geometries that are unknown in advance,

- it is optimized with respect to structural as well as geometrical criteria.

In contrast to other research on simultaneous shape and sizing optimization of structures (Kociecki \& Adeli, 2015, Li. W., Pu, H., Schonfeld, P., Song, Z., Zhang, H., Wang, L., Wang, J., Peng, X., \& Peng, L. 2018), the approach proposed here focuses on the local shape/geometry of the basic module, instead of the global shape of the entire structure. Moreover, it includes an additional objective function in a multiobjective optimization approach in order to assess the geometrical versatility of the resulting modular system simultaneously with its structural performance.

In computational terms, the proposed optimization procedure has a nontrivial two-level character. The upper-level optimization variables are the geometric parameters that define the shape of the module. The geometric and the structural objective functions are computed at this level and balanced by means of Pareto front. The geometric objective function is computed directly by generating and assessing possible global configurations. The computation of the structural objective function requires a separate lower-level optimization process with respect to structural parameters of the module and subject to structural design constraints. These constraints take here the form of upper bounds imposed on the von Mises stress and the critical buckling load ratio. This lower-level optimization is performed using an adaptive-neighborhood variant of the simulated annealing algorithm, in which the global temperature governs not only the transition probability of the search point, but also the definition of its neighborhood: such an approach is intended to intensify the exploration in the vicinity of the constraints that are expected to be active at the optimum. The upper-level optimization amounts to determination of the Pareto front, and it is performed using the non-dominated sorting genetic algorithm II, NSGA-II (Deb, Pratap, Agarwal, \& Meyarivan, 2002), which is one of the multiobjective evolutionary algorithms (A. Zhou et al., 2011).

The paper is organized as follows: Section 2 introduces the specific modular system considered here. The geometry of the module and the optimization variables are discussed in Section 3 The assessment criteria (objective functions) are proposed in Section 4, and the computational aspects are discussed in Section 5 Finally, Section 6 presents the optimization results.

\section{2 | THE CONCEPT OF TRUSS-Z}

Truss-Z (TZ) is a skeletal modular ramp system based on frame modules (TZMs) and intended for pedestrian traffic.

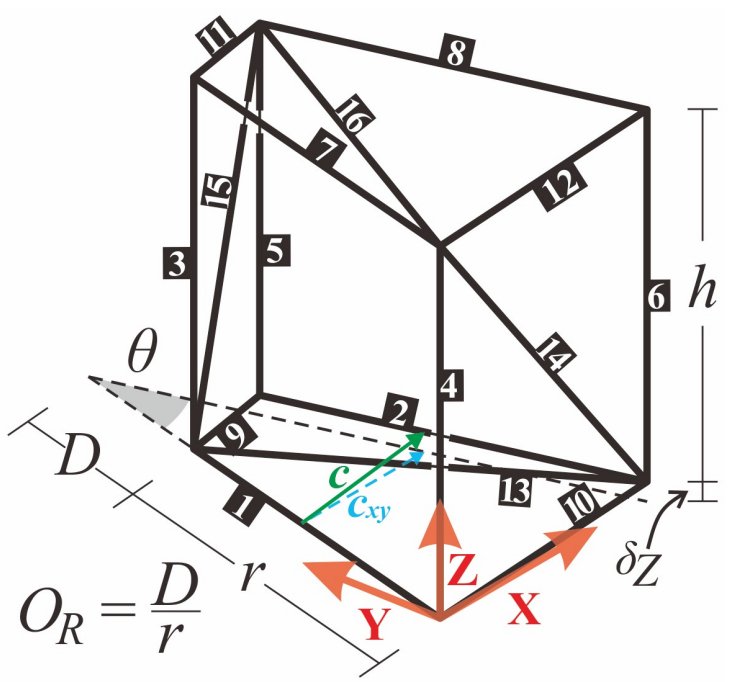

FIGURE 1 The geometry of the original Truss-Z module R.

It is an example of an Extremely Modular System: a system designed for creation of free-form shapes (serving given purposes) with the use of as few types of modules as possible. Truss- $Z$ is intended for pedestrian traffic and it has been introduced to improve the mobility of the elderly, wheelchair users and people with baby-strollers by simple and affordable means. It is designed to be retrofittable and minimally invasive, but most importantly, due to the extreme modularization - economically competitive.

\section{1 | TZ modules}

All TZ structures are assembled of four variations of a single basic module. The module is an adaptation of the basic section of a conventional truss bridge, which enables curved layout design and variation in the inclination of a sequence of modules. The basic module is denoted by R, which stands for "right" as, according to the right-hand grip rule, it "turns left and goes up". The geometry of the original R used in previous research has been decided arbitrarily. It is illustrated in Figure 1 for an explanation of the involved symbols see Section 3.1. Consequently, the module variation $\mathrm{L}$ can be denoted as "right and up" and it is the mirror reflection of the module $\mathrm{R}$. In practice, $\mathrm{R}$ and $\mathrm{L}$ need to be fabricated independently. The next two variations come from a simple rotation by $\pi / 2$ about the vertical axis: $R_{2}$ and $L_{2}$ are the rotated $R$ and $\mathrm{L}$, respectively. Figure 2 shows examples of simple TZ structures composed of these four variations and demonstrates the versatility of this system. The modules have been fabricated with glued together wooden bars and painted for illustrative purposes. To simplify the fabrication, the bars overlap which results in eccentricity of joints. 


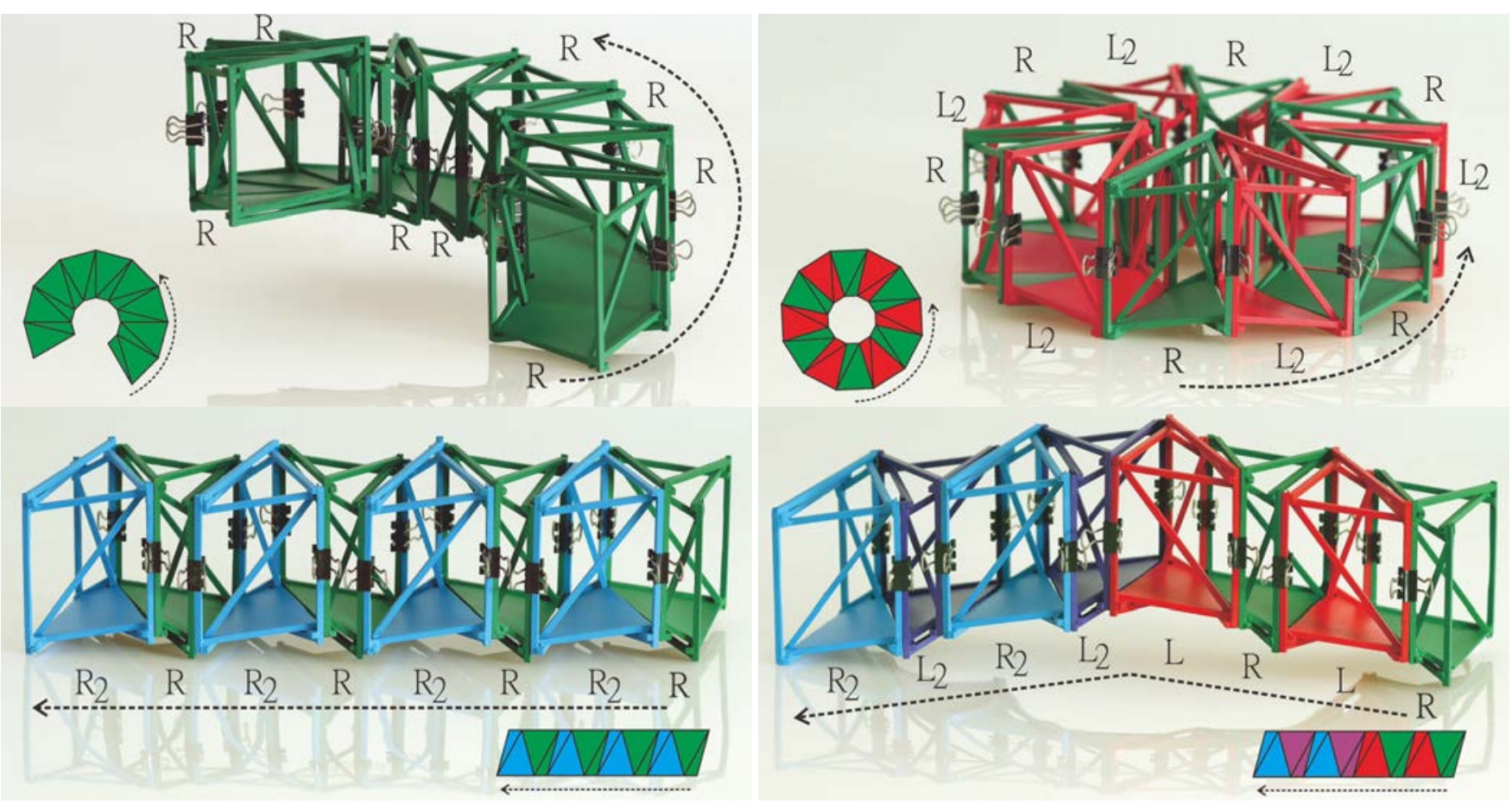

FIGURE 2 Photographs of the early models of TZ structures. The modules made of glued together wooden bars are assembled with binder clips for quick and firm connection. From top left to bottom right: A TZ spiral with 12 repeated modules R; A TZ flat ring constructed with 12 alternating modules: $\mathrm{R}$ and $\mathrm{L}_{2} ; \mathrm{An} 8$-module straight and flat $\mathrm{TZ}$ composed of repeated pairs $\mathrm{RR}_{2}$; Straight up and down TZ with 8 modules: RLRLL ${ }_{2} \mathrm{R}_{2} \mathrm{~L}_{2} \mathrm{R}_{2}$. A schematic top view of the configuration shown at each photograph. The module variations: $R, L, R_{2}$ and $L_{2}$ are shown in: green, red, cyan and magenta, respectively.

Figure 3 shows a photograph that illustrates the case study described in Zawidzki(2015), where TZ has been proposed for retrofitting of an existing footbridge connecting two sections of a university campus that are separated by a four-lane street. The existing footbridge is highly inaccessible, as, for example, one of the stairways is very long and particularly steep: 29 high risers without any intermediate landing.

\subsection{Global geometry of Truss-Z structures}

Due to its modular nature, designing a path of a TZ structure between two or more points is a constrained discrete optimization problem. The most straightforward and natural objective is the minimization of the number of modules (TZMs), which expresses the economization of construction. Other possible objectives considered in earlier research (Zawidzki. 2015) are the number of TZMs pairs which do not create a straight line and the number of continuous turns in a TZ path. In natural language, these objectives can be respectively explained as: users prefer to make as few turns as possible and it is preferable to make fewer but longer continuous turns rather than a larger number of shorter turns. In the case of a multi-branch
$\mathrm{TZ}$, it is natural to minimize its network distance as proposed in Zawidzki (2016).

The most obvious constraint in the process of TZ path design is the location of the points to be linked by the structure. Further natural constraints are the prohibition of self-collisions among the TZMs and the prohibition of collisions with the obstacles present in the environment, such as buildings and street lights. Other practical constraints (Zawidzki \& Szklarski) 2018) might be the maximum allowable span of an unsupported TZ assembly, minimization of earthworks, preservation or minimal removal of the existing trees.

As discussed in Zawidzki (2015), various deterministic and meta-heuristic computational methods have been successfully applied for the geometric design of single-branch TZ paths, including backtracking and evolutionary algorithms. Image processing methods parallelized with GPU have been implemented for effective TZ layout optimization in Zawidzki \& Szklarski (2018). For optimization of multi-branch TZ layouts, evolutionary algorithms have been successfully applied in Zawidzki (2016). A graph-theoretic exhaustive search for TZ optimization, which produced the best allowable, that is the ideal solutions, has been presented in Zawidzki (2015). 


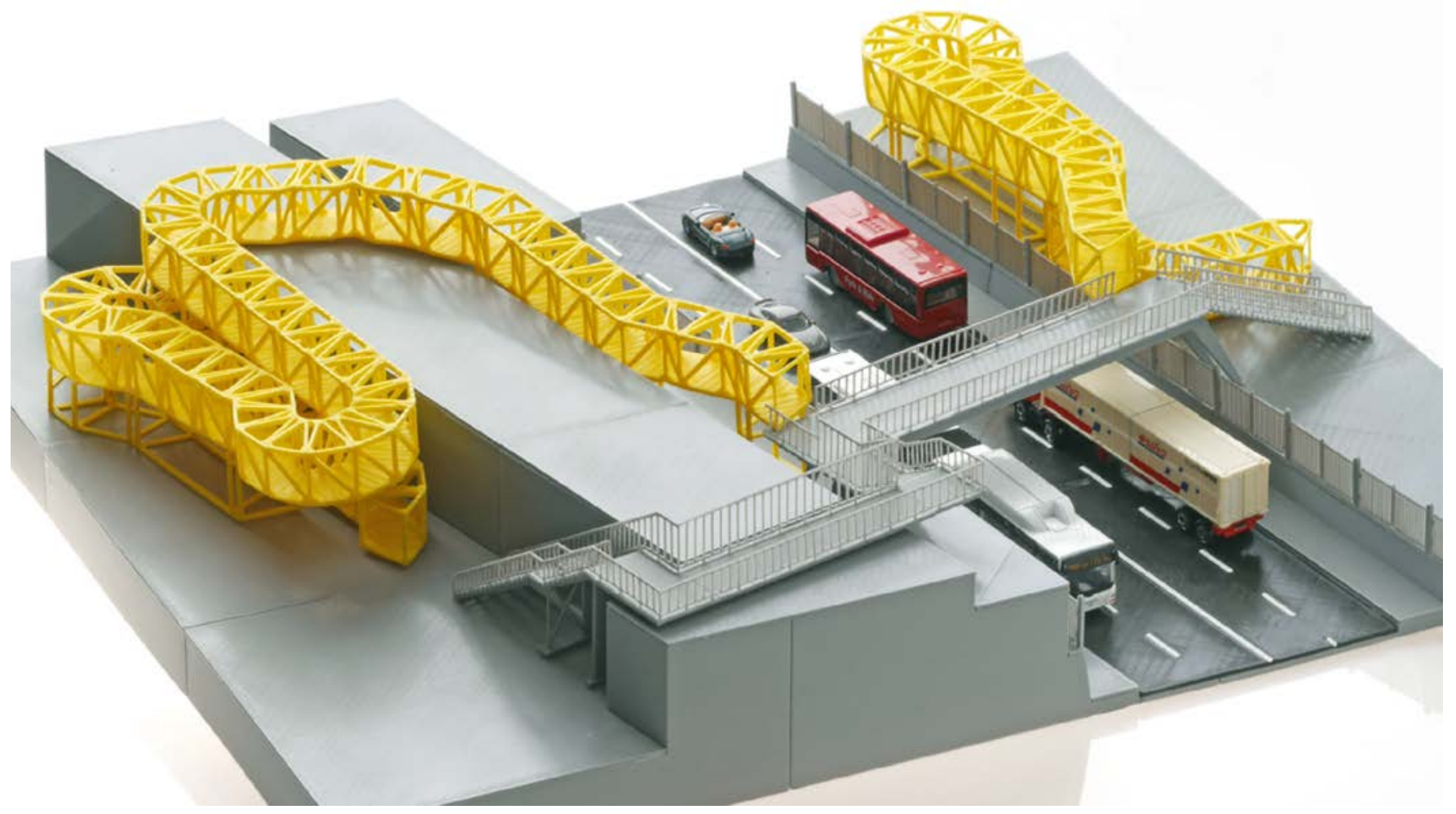

FIGURE 3 A photograph of a reduced-scale physical model. It shows an existing highly inaccessible footbridge at Hongo Campus of the University of Tokyo retrofitted with Truss-Z (shown in yellow). This would allow for wheelchair mobility, as the slope of the TZ is approximately 1:12 (ADA Standards for Accessible Design, 2010).

\subsection{Local module design and this work}

All the aforementioned research on TZ considered only the problems related to the global geometric layout. In Zawidzki \& Jankowski(2018), a particular outer geometry of the module is arbitrarily assumed, and the problem of sizing optimization of TZM members is considered. The first attempt at multicriterial optimization of a TZM for a single-branch TZs is presented in a conference report Zawidzki \& Jankowski (2017), where a relatively simpler and more specific optimization problem is considered: a module of a constant mass is used, buckling is neglected and the geometric objective is expressed in terms of TZ suitability for a single particular construction site. In contrast, this research attempts to answer the following, more general question: What is the structurally and functionally optimal universal TZM? In other words, the aim here is to optimally design a TZM not for a specific, particular spatial environment, but rather for all possible application scenarios.

\section{3 | TRUSS-Z MODULE}

This section first introduces the geometry of the TZ module and then defines its geometric and structural parameters that are used as the optimization variables.

\section{1 | TZ module geometry}

The geometry of the module is determined by the following parameters: planar angle $\theta$, offset ratio $O_{R}$, width $r$, height $h$ and rise $\delta_{\mathrm{Z}}$, see Fig. 1 The floor center-line vector is denoted by $\mathbf{c}$, while $c_{\mathrm{xy}}$ is the length of its projection onto the horizontal plane xy. The offset ratio $O_{R}$ is defined as the ratio of the offset $D$ from the apex to the module width $r$. For constant width $r$ and length $c_{\mathrm{xy}}$, the offset ratio $O_{R}$ controls the shape of the module projection onto the xy plane, which for the cases $O_{R}=0,0<O_{R}<\infty$ and $O_{R}=\infty$ is respectively a triangle, a trapezoid, and a rectangle. The offset ratio is related to the planar angle, width and length parameters as follows:

$$
c_{\mathrm{xy}}=2 r\left(O_{R}+\frac{1}{2}\right) \sin \frac{\theta}{2} .
$$

In earlier research on TZ structures, a module of an arbitrarily assumed geometry has been used, shown in Fig. 1 This 
original module is defined as follows:

$$
\begin{aligned}
& \theta_{0}=30^{\circ}, \quad r_{0}=2.4 \mathrm{~m}, \quad O_{R 0}=0.5, \\
& \delta_{\mathrm{Z} 0}=0.1 \mathrm{~m}, \quad h_{0}=2.4 \mathrm{~m}, \quad c_{\mathrm{xy} 0}=1.242 \mathrm{~m} .
\end{aligned}
$$

The height $h_{0}$ and the width $r_{0}$ of the entrance and exit frames $(2.4 \mathrm{~m} \times 2.4 \mathrm{~m})$ have been selected based on functional requirements. The rise $\delta_{\mathrm{Z} 0}$ has been selected to keep the center-line inclination $\delta_{\mathrm{Z} 0} / c_{\mathrm{xy} 0}$ within the maximum acceptable ramp slope of 1:12 (ADA Standards for Accessible Design 2010). The specific configuration of the diagonal beams shown in Fig. 1 has been obtained in Zawidzki \& Jankowski (2018) and retained in this paper.

\subsection{Geometry-related optimization variables}

Two geometric parameters of the module are selected for the purpose of shape optimization:

1. the planar angle $\theta$ and

2. the center-line projection length $c_{\mathrm{xy}}$.

These two parameters are collected in the vector $\mathbf{x}$,

$$
\mathbf{x}=\left(\theta, c_{\mathrm{xy}}\right),
$$

and considered to vary within the following search domain:

$$
\begin{aligned}
& \theta \in\left[15^{\circ}, 45^{\circ}\right], \\
& c_{\mathrm{xy}} \in\left[0.8 c_{\mathrm{xy} 0}, 2.0 c_{\mathrm{xy} 0}\right] \approx[1.0 \mathrm{~m}, 2.5 \mathrm{~m}] .
\end{aligned}
$$

The other geometric parameters of the module are not optimized: they are treated as uniquely determined by the optimization variables $\theta$ and $c_{\mathrm{xy}}$, as well as the functional requirements:

- The entrance frame and the exit frame retain the original dimensions of $r=2.4 \mathrm{~m}$ and $h=2.4 \mathrm{~m}$.

- Given the width $r$ and the optimization variables $\theta$ and $c_{\mathrm{xy}}$, the offset ratio $O_{R}$ is determined by 1 .

- The rise $\delta_{\mathrm{Z}}$ is determined by fixing the inclination of the center-line vector at the same acceptable maximum value as in the original module (ADA Standards for Accessible Design, 2010),

$$
\delta_{\mathrm{Z}}=c_{\mathrm{xy}} \frac{\delta_{\mathrm{Z} 0}}{c_{\mathrm{xy} 0}} .
$$

\section{3 | Structural optimization variables}

A spatial frame model is used for the module. The beams are modeled as thin-walled circular hollow sections (CHS) with a constant wall thickness $d=2 \mathrm{~mm}$. The typical material parameters of steel are assumed: the density $7800 \mathrm{~kg} / \mathrm{m}^{3}$, Young's modulus $205 \mathrm{GPa}$ and the shear modulus $79.3 \mathrm{GPa}$.
A sizing optimization problem is considered, in which the structural optimization variables represent the diameters $y_{i}$ of the 16 module beams. They are collected in the vector $\mathbf{y}$,

$$
\mathbf{y}=\left(y_{1}, y_{2}, \ldots, y_{16}\right) \text {. }
$$

Taking into account the wall thickness $d=2 \mathrm{~mm}$, the parameters are subjected to the following natural lower bound constraint:

$$
y_{i} \geq 2 d=4 \mathrm{~mm} \quad \text { for } i=1,2, \ldots, 16 .
$$

\section{4 | ASSESSMENT CRITERIA}

Essentially, designing a TZ module is a multicriterial optimization problem (Ehrgott, 2005; Marler \& Arora, 2004). The solution process proposed here aims to balance between the two following, intrinsically very different types of objectives:

1. The ability of the module to generate a variety of freeform shaped global TZ structures. This is quantified by assessing the directionality of the exit modules and the spatial distribution of their end points, which are required to be possibly uniform. The aim is to promote systems that are flexible enough to comply with intricate geometrical constraints of real construction sites.

2. The structural quality of the generated global TZ structures. Notwithstanding the differences in formulation, it can be expressed in analogy to a structural optimization problem, in which mass is minimized subject to constraints that prevent yielding and buckling.

This section introduces the criteria used to assess a TZ module in geometric and structural terms, including the related constraints.

\section{1 | Assessment of geometrical quality}

A Truss- $Z$ structure is constructed as an assembly of modules that link the entrance point and the exit point. A geometrically versatile module should be able to generate TZs that

- reach points possibly uniformly distributed in the neighborhood of the entrance module, that is without significant gaps and clusters (see Section 4.1.1). Moreover,

- each of these exit points should be reachable from different (possibly uniformly distributed) directions of the exit module (see Section 4.1.2).

As an example, Fig. 4 shows (projections onto the horizontal plane of) a few selected TZs of 24-unit length which can be generated from a single starting module that is drawn bold; the entrance point and the exit points are explicitly marked, 


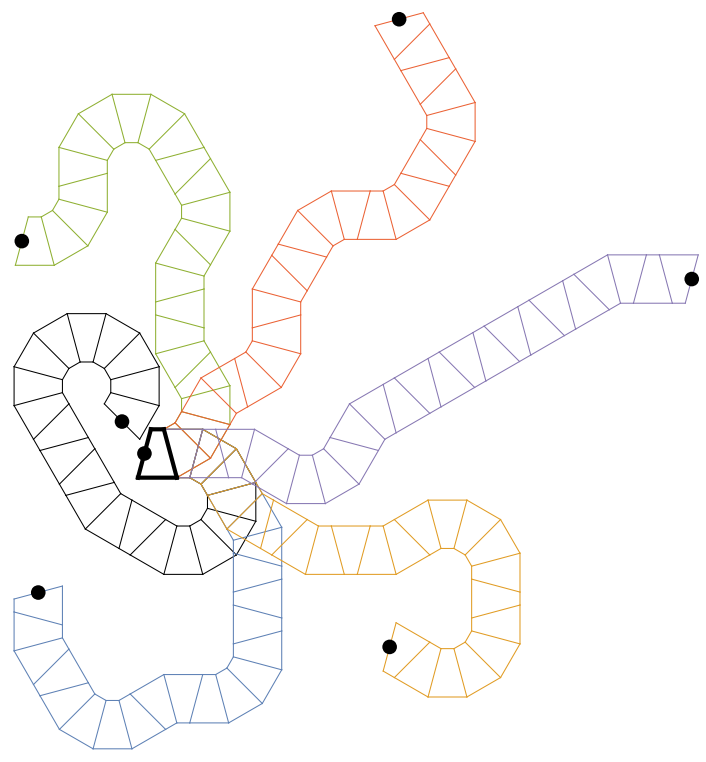

FIGURE 4 Projections onto the horizontal plane of a few selected TZs that can be generated from a single starting module (drawn in bold). The entrance and the exit points are marked with black dots.

and the module is assumed to have the geometry defined by $\mathbf{x}=\left(30^{\circ}, c_{\mathrm{xy} 0}\right)$. Note that the $\mathrm{TZ}$ drawn in black cannot be prolonged much further due to the imminent self-intersection.

The following two subsections define two objective functions, $I_{\mathrm{xy}}(\mathbf{x})$ and $I_{\alpha}(\mathbf{x})$, that quantify the spatial and angular uniformity of the exit modules. In Section 6 they are verified to be highly correlated and thus effectively constitute equivalent measures of the geometric versatility.

\subsection{1 | Distribution of $\mathbf{T Z}$ exit points}

Consider a single module (either R or L), placed at the origin and facing a certain direction. Consider also all the TZs that start with this module and that are each composed of no more than $n$ modules that do not collide with each other (the modules are of a finite width, and thus the self-intersection check has to involve the perimeters of the modules instead of their centerlines). The (projections onto the xy plane of the) exit points of all these TZs form a point cloud, which depends on the geometric parameters $\mathbf{x}$ of the module. Quantitative analysis (although not optimization) of such spatial point patterns is a typical problem in geographic information systems (GIS), see for example Illian, Penttinen, Stoyan, \& Stoyan (2008) or Chapter 8 in Cressie (2015). In the considered case, the point cloud should possibly uniformly cover the neighborhood of the starting point. The typical quantitative measures used for this task are based on the point distances to their nearest neighbors, and they are designed to assess random patterns. For geometrically structured patterns considered here, which naturally

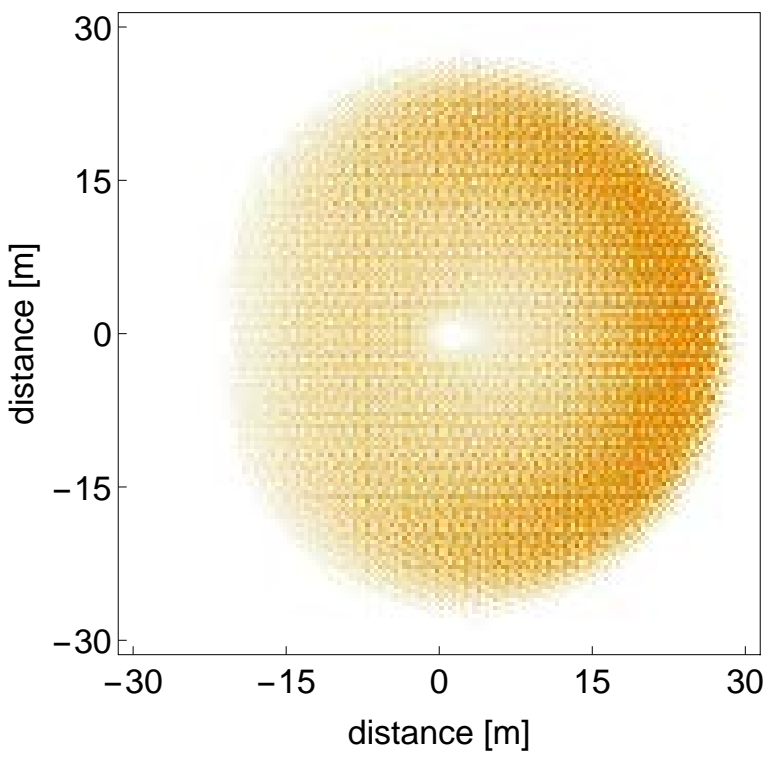

FIGURE 5 The point counts generated by all the TZs up to 24 module long on a rectangular grid of $40 \mathrm{~cm} \times 40 \mathrm{~cm}$ bins.

feature many overlapping points, a better-suited approach is offered by the quadrat methods, which first divide the reachable space into $N$ subregions (quadrats or bins) and then represent and assess the point pattern via the counts $p_{i}, i=1,2, \ldots, N$, of the points falling into these subregions. Figure 5 shows the point counts that correspond to Fig. 4 and are generated by all possible TZs up to 24 module long; the bins are $40 \mathrm{~cm} \times 40 \mathrm{~cm}$, which is a typical space occupied by a human agent in crowd simulations (Feliciani \& Nishinari 2016, Weidmann, 1993). The point cloud is almost symmetric, and its density tends to increase to the boundary, which result from the directionality of the initial module that guides the growth of possible paths into the initial direction (to the right in the example of Fig. 5 .

The ability of a module to generate a possibly uniform coverage of the neighborhood can be explicitly quantified in terms of the index of dispersion of the point counts (Cressie, 2015, Illian et al. 2008), which is a normalized quantitative regularity measure of point patterns. It is based on the $\chi^{2}$ goodness of fit statistic and defined as the ratio of the sample variance of the point counts to their mean,

$$
I_{\mathrm{xy}}(\mathbf{x})=\frac{s_{p}^{2}}{\bar{p}},
$$

where $s_{p}^{2}$ is the sample variance of the point counts and $\bar{p}$ is the mean number of points per bin,

$$
\begin{aligned}
& \bar{p}=\frac{1}{N} \sum_{i=1}^{N} p_{i}, \\
& s_{p}^{2}=\frac{1}{N-1} \sum_{i=1}^{N}\left(p_{i}-\bar{p}\right)^{2} .
\end{aligned}
$$


A small value of the objective function $I_{\mathrm{xy}}(\mathbf{x})$ indicates spatial uniformity (or regularity) of the point pattern, while a large value indicates nonuniformity or clustering of the points: the lower the index, the more uniformly the exit points are distributed. Note that even with a moderate number $n$ of modules, evaluation of the respective point cloud is a computationally intensive operation, as it requires an exponentially growing number $O\left(2^{n}\right)$ of TZs to be generated and verified for self-intersections.

\subsection{2 । Local directionality of the exit modules}

Versatility of the module geometry should be assessed also in terms of the angular distribution of the directions from which the $i$ th bin can be attained. Denote thus by $\alpha_{j i}, j=1,2, \ldots, p_{i}$, the angular orientations of the modules with the exit points within the $i$ th bin. The angular uniformity of these directions can be quantified in terms of the Kolmogorov-Smirnov (KS) statistic $D_{i} \sqrt{p_{i}}$. The value $D_{i}$ is defined as the maximum deviation of the empirical distribution function from the cumulative distribution function,

$$
D_{i}=\sup _{\alpha \in[0,2 \pi]}\left|F_{i}(\alpha)-\frac{\alpha}{2 \pi}\right|,
$$

where $\alpha / 2 \pi$ is the cumulative distribution function of the uniform angular distribution and $F_{i}(\alpha)$ is the empirical distribution function computed within the $i$ th bin for the module orientations $\alpha_{j i}, j=1,2, \ldots, p_{i}$,

$$
F_{i}(\alpha)=\frac{1}{p_{i}} \sum_{j=1}^{p_{i}} \mathrm{q}_{[0, \alpha]}\left(\alpha_{j i}\right),
$$

where $\mathbb{1}_{[0, \alpha]}\left(\alpha_{j i}\right)$ is the indicator function

$$
\mathbb{\square}_{[0, \alpha]}\left(\alpha_{j i}\right)=\left\{\begin{array}{l}
1 \text { if } \alpha_{j i} \leq \alpha, \\
0 \text { otherwise. }
\end{array}\right.
$$

The smaller $D_{i}$, the more uniform are the empirical distribution and the local angular orientations of the exit modules within the $i$ th bin.

The following objective function is proposed to aggregate the local statistics $D_{i}$ at the global level in terms of their root mean square value:

$$
I_{\alpha}(\mathbf{x})=\operatorname{rms}_{i}\left(D_{i}(\mathbf{x}) \sqrt{p_{i}(\mathbf{x})}\right) .
$$

Multiplication by $\sqrt{p_{i}}$ is required to account for different values of the bin counts $p_{i}$. A small value of $I_{\alpha}(\mathbf{x})$ indicates the domination of bins with the intended good angular uniformity of the exit modules.

\section{2 | Assessment of structural quality}

The structural quality of a TZ module can be expressed in general terms as its ability to generate lightweight, free-form shaped TZ structures capable of carrying a certain design load. This is a vague formulation, which is formalized here in analogy to the classic minimum weight problem (Gilbert \& Tyas, 2003, Sokót 2011, Zegard \& Paulino, 2015). The problem considered here differs significantly from the typical formulation in several details, which require a specific approach:

- The module is not a truss with a simple uniaxial stress state, but rather a frame structure with a more complex stress state. Therefore, two types of constraints are used: (i) an upper bound constraint imposed on the von Mises stress to prevent yielding and (ii) an upper bound constraint imposed on the compressive force of each beam to prevent buckling. Both constraints are computed under a certain static design load $\mathbf{P}$.

- Each structural element is not a simple bar, but rather a beam of a circular hollow profile.

- The outer geometry of the module (including its length) is variable, so that the mass of the module and the stress state depend not only on the beam cross-sections $\mathbf{y}$, but also on the geometric parameters $\mathbf{x}$.

- A modular structure is considered, that is (i) several identical copies of the module are used in various localizations within the global structure, and (ii) several global structures of different geometries need to be considered, see Fig. 2 for simple illustrative examples.

- The variable geometry of the module affects the shape and the length of the resulting global TZ structures. The static design load $\mathbf{P}$ cannot be thus assumed to be constant: in order to model crowd loads, it needs to depend on the module area (the geometric parameters $\mathbf{x}$ ).

\subsection{1 | Stress constraint}

For a TZ that corresponds to a given geometric configuration $s$ of modules, the stress state is quantified by means of the maximum effective stress. Let $\sigma_{i n s}^{\max }(\mathbf{x}, \mathbf{y})$ denote the maximum von Mises stress of the $i$ th beam in the $n$th module of the configuration $s$ that occurs under a given static load vector $\mathbf{P}_{s}(\mathbf{x})$. The considered system is modular, so that structural assessment needs to be performed not for a single particular configuration $s$ of the modules, but rather for a set $S$ of all possible configurations. The worst-possible von Mises stress is considered separately for each beam $i$ and subjected to the following upper bound constraint:

$$
\rho_{i}^{\sigma}(\mathbf{x}, \mathbf{y})=\frac{\max _{s \in S} \max _{1 \leq n \leq \bar{s}} \sigma_{i n s}^{\max }(\mathbf{x}, \mathbf{y})}{\sigma^{\max }} \leq 1,
$$


where $i \in\{1,2, \ldots, 16\}$ indexes the beams, $\bar{s}$ denotes the number of modules in the configuration $s$, and

$$
\sigma^{\max }=100 \mathrm{MPa}
$$

is an arbitrary value assumed here to be safe for steel.

\subsection{2 | Compressive force constraint}

Let $f_{\text {ins }}(\mathbf{x}, \mathbf{y})$ denote the compressive force of the $i$ th beam in the $n$th module of the configuration $s$ under the static design load $\mathbf{P}_{s}(\mathbf{x})$. In an analogy to the stress constraint, the risk of buckling is limited locally for each beam by imposing the following upper bound on the worst-case compressive force:

$$
\rho_{i}^{\mathrm{f}}(\mathbf{x}, \mathbf{y})=\frac{\max _{s \in S} \max _{1 \leq n \leq \bar{s}} f_{i n s}^{\max }(\mathbf{x}, \mathbf{y})}{0.85 f_{i}^{\text {Euler }}(\mathbf{x}, \mathbf{y})} \leq 1 .
$$

The coefficient 0.85 is the safety factor and $f_{i}^{\text {Euler }}(\mathbf{x}, \mathbf{y})$ is Euler's critical load of the $i$ th beam,

$$
f_{i}^{\text {Euler }}(\mathbf{x}, \mathbf{y})=\pi^{2} \frac{E I_{i}(\mathbf{x}, \mathbf{y})}{l_{i}^{2}(\mathbf{x})},
$$

where $E I_{i}(\mathbf{x}, \mathbf{y})$ and $l_{i}(\mathbf{x})$ denote respectively the bending stiffness and the length of the $i$ th beam.

\subsection{3 | Module mass per unit length}

The structural objective of optimization is to minimize the mass of the module per its unit length in the xy plane,

$$
m(\mathbf{x}, \mathbf{y})=\frac{\pi d \sum_{i=1}^{16}\left(y_{i}-d\right) l_{i}(\mathbf{x})}{c_{\mathrm{xy}}},
$$

where $l_{i}$ denotes the length of the $i$ th beam of the module. As defined in Section 3.3, $y_{i}$ are the elements of the vector $\mathbf{y}$ and denote the diameters of the beams, which are circular hollow section profiles with a constant wall thickness $d=2 \mathrm{~mm}$.

Notice that (11) depends on the module geometry $\mathbf{x}$, as well as on the diameters $\mathbf{y}$ of its beams, while the geometric objective function (8) depends on the geometry $\mathbf{x}$ only. This suggests that, for each specific geometry $\mathbf{x}$, the module can be first optimized with respect to $\mathbf{y}$ in an inner-loop optimization, subject to the lower bound constraints $(5 \mathrm{~b})$ and the design constraints (9a) and (10a). The proposed objective function quantifies thus the mass of the mass-minimum module and depends only on the geometric parameters $\mathbf{x}$,

$$
\begin{aligned}
m(\mathbf{x})= & \min _{\mathbf{y}} m(\mathbf{x}, \mathbf{y}) \\
& \text { subject to } 5 \mathrm{~b}, \text {, 9a and } 10 \mathrm{a}) .
\end{aligned}
$$

In the proposed formulation, the subject to minimization is the mass per unit length of the module. It differs from the objective typically used in structural optimization, which is minimization of the total mass. The reason is that the mass of the TZM depends not only on the beam diameters $\mathbf{y}$, but also

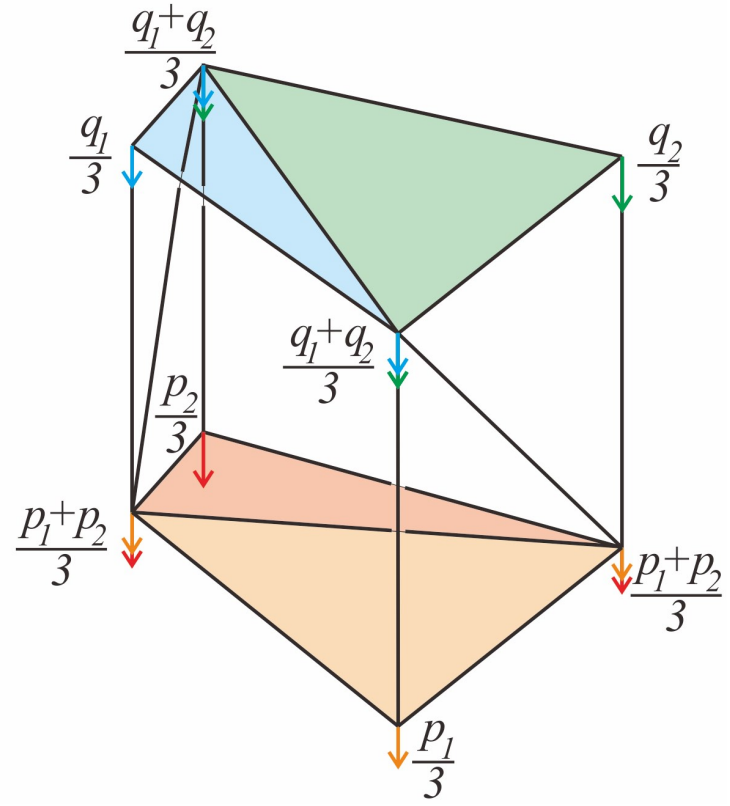

FIGURE 6 The static design load $\mathbf{P}_{s}(\mathbf{x})$ of a single TZM used for optimization purposes. The floor and the ceiling area loads $\left(4000 \mathrm{~N} / \mathrm{m}^{2}\right.$ and $1000 \mathrm{~N} / \mathrm{m}^{2}$ ) are each distributed to the two congruent triangles ( $p_{1}$ and $p_{2}, q_{1}$ and $q_{2}$ ), which are treated as rigid and distribute their loads to the respective endpoints of the beams.

on their lengths defined by the geometric variables $\mathbf{x}$. Therefore, unlike in Zawidzki \& Jankowski (2018), the length of the module needs to be accounted for; otherwise the optimization will improperly prefer shorter modules not because of their purported structural superiority, but only because they have shorter beams and thus a smaller mass.

\subsubsection{Static design load and boundary conditions}

The design constraints (9a) and (10a) impose a limit on the effective stresses and compressive forces in all possible Truss$\mathrm{Z}$ configurations $s \in S$, each subjected to the respective static design load $\mathbf{P}_{s}(\mathbf{x})$. To compute the effective stresses for the optimization purposes, a vertical patterned area load of $5000 \mathrm{~N} / \mathrm{m}^{2}$ is used, which slightly exceeds the value of $90 \mathrm{psf}$ $\left(4309 \mathrm{~N} / \mathrm{m}^{2}\right)$ suggested for pedestrian loading by the LRFD Guide Specification for the Design of Pedestrian Bridges (2009). The load is distributed between the floor and the ceiling of the TZ in proportion 4:1. On each level, it is allocated to the endpoints of the horizontal beams, proportionally to the area of the beam triangles that constitute the floor and the ceiling. Figure 6 illustrates the load allocation scheme on the example of a single module of the original dimensions. The floor and 
the ceiling are each composed of two triangles, which mimic rigid panels and which are subjected respectively to the vertical loads $p_{1}$ and $p_{2}$ or $q_{1}$ and $q_{2}$, in proportion to their area. The assumption of rigidity of the triangle panels allows these loads to be evenly distributed among the vertices (endpoints of the respective beams), which results in the loading pattern shown in Fig. 6

For each considered TZ configuration $s \in S$, each of its component modules is separately subjected to the load defined above, and then all the resulting module loads are assembled into the global load vector $\mathbf{P}_{s}(\mathbf{x})$ of the entire TZ structure. In order to compute the stresses and compressive forces, the considered $\mathrm{TZ}$ configurations are treated as supported at the beginning and at the end, that is fixed supports are assumed in the entrance of the first module and in the exit of the last module.

\section{5 | COMPUTATIONAL ASPECTS}

The considered optimization problem can be stated as follows: minimize simultaneously the following objective functions:

- $I_{\mathrm{xy}}(\mathbf{x})$ and $I_{\alpha}(\mathbf{x})$, defined in 6 a and $(8)$, which express the geometric versatility of the module, and

- $m(\mathbf{x})$, defined in $(12)$, which is related to the structural performance of the module,

in a Pareto-type multicriterial optimization, with respect to the geometry-related variables $\mathbf{x}$ and within the domain defined in (3b).

Despite the seemingly simple formulation, the proposed optimization problem has a nontrivial two-level character and is computationally relatively demanding. The lower level provides the values of the considered objective functions, which involves an inner-loop structural optimization procedure, while the upper level aggregates the provided values into the multiobjective optimization problem and determines the Pareto front.

\section{1 | Structural objective function}

The objective function (12) is computationally expensive: it involves a complex inner-loop structural optimization problem of the sizing type that has to be solved simultaneously for several structures, subject to the maximum effective stress and compressive force constraints, (9a) and (10a), and the lower bound constraint $(5 \mathrm{~b})$. The optimization variables are the structural parameters $\mathbf{y}$ of the module, and the optimum point $\overline{\mathbf{y}}$ depends on its geometry, that is on the vector $\mathbf{x}$ of the geometric parameters.
Notice that this inner-loop optimization is performed simultaneously with respect to a large number of global structures. It is assumed here that the longest unsupported span in a TZ structure is composed of not more than five modules. Computation of the constraints (9a) and (10a) requires thus to analyze the set $S$ of all possible spatial configurations of five TZ modules, $\bar{s}=5$. Each module in such a five-module $\mathrm{TZ}$ can be used in one of the four possible variations $\left(\mathrm{R}, \mathrm{L}, \mathrm{R}_{2}\right.$ or $\mathrm{L}_{2}$, see Section 2). There are $4^{5}=1024$ such structures, but if the rotation $\left(\mathrm{R}-\mathrm{R}_{2}, \mathrm{~L}-\mathrm{L}_{2}\right)$, the left-right symmetry $\left(\mathrm{R}-\mathrm{L}, \mathrm{R}_{2}-\right.$ $\mathrm{L}_{2}$ ) and the front-back symmetry $\left(\mathrm{R}-\mathrm{L}_{2}, \mathrm{~L}-\mathrm{R}_{2}\right)$ are taken into account, the set $S$ reduces fourfold to 256 essentially unique configurations. At each iteration of the inner-loop optimization, all these configurations need to be subjected to the load $\mathbf{P}_{s}(\mathbf{x})$ and independently analyzed to compute the constraint functions $\rho_{i}^{\sigma}(\mathbf{x}, \mathbf{y})$ and $\rho_{i}^{\mathrm{f}}(\mathbf{x}, \mathbf{y})$.

The inner-loop optimization is performed using an adaptive variant of the simulated annealing algorithm, in which the global temperature governs not only the transition probability of the search point, but also the probabilistic definition of its neighborhood. At high system temperatures, the neighborhood is symmetric around the current search point $\mathbf{y}$. However, when the system temperature decreases, it shifts towards a rough estimation $\overline{\mathbf{y}}$ of the optimum, defined as a point with design constraints active for all beams. More specifically, in each iteration, the neighbor of the point $\mathbf{y}$ is drawn from the probability distribution

$$
N\left(\mathbf{y}+\frac{T_{0}-T}{T_{0}} \Delta \mathbf{y}, \zeta \operatorname{diag}|\Delta \mathbf{y}|\right),
$$

where $T$ is the current system temperature, $T_{0}$ is the initial temperature, $\Delta \mathbf{y}=\overline{\mathbf{y}}-\mathbf{y}$ is the vector that points from the current search point $\mathbf{y}$ to the estimated optimum $\overline{\mathbf{y}}$, and diag $|\Delta \mathbf{y}|$ denotes the diagonal matrix with $|\Delta \mathbf{y}|$ on the diagonal. Such an approach gradually intensifies the exploration in the vicinity of the constraints that are expected to be active at the optimum, and it belongs to the general class of simulated annealing algorithms with adaptive neighborhood (Xinchao 2011).

The rough estimation $\overline{\mathbf{y}}$ of the optimum is obtained as follows. For each beam, three different constraints are formulated: stress constraint $9 \mathrm{a}$, , compressive force constraint (10a) and lower bound constraint $(5 \mathrm{~b})$, of which the first two are design constraints. Therefore, at the optimum, by an analogy to the classical fully stressed design (Patnaik \& Hopkins 1998), one of them can be expected to be active for each beam, at least unless the lower bound constraint $(5 \mathrm{~b})$ is activated. It can be thus supposed that the optimum point $\overline{\mathbf{y}}$ satisfies the equation

$$
\max \left(\rho_{i}^{\sigma}(\mathbf{x}, \overline{\mathbf{y}}), \rho_{i}^{\mathrm{f}}(\mathbf{x}, \overline{\mathbf{y}})\right)=1 \quad \text { or } \quad y_{i}=2 d
$$

for all $i=1,2, \ldots, 16$. For a given vector $\mathbf{y}$ of beam diameters (the current search point), the solution to (14) can be roughly 
estimated by assuming that

$$
\begin{aligned}
\rho_{i}^{\sigma}(\mathbf{x}, \mathbf{y}+\Delta \mathbf{y}) & \sim \frac{1}{A\left(y_{i}+\Delta y_{i}\right)}, \\
\rho_{i}^{\mathrm{f}}(\mathbf{x}, \mathbf{y}+\Delta \mathbf{y}) & \sim \frac{1}{E I_{i}\left(y_{i}+\Delta y_{i}\right)},
\end{aligned}
$$

which amounts to an assumption that all internal stresses are inversely proportional to the beam cross-section area $A\left(y_{i}\right)$ and that the internal forces are independent of $\Delta \mathbf{y}$. Consequently,

$$
\begin{aligned}
\rho_{i}^{\sigma}(\mathbf{x}, \mathbf{y}+\Delta \mathbf{y}) & \approx \frac{A\left(y_{i}\right)}{A\left(y_{i}+\Delta y_{i}\right)} \rho_{i}^{\sigma}(\mathbf{x}, \mathbf{y}), \\
\rho_{i}^{\mathrm{f}}(\mathbf{x}, \mathbf{y}+\Delta \mathbf{y}) & \approx \frac{E I_{i}\left(y_{i}\right)}{E I_{i}\left(y_{i}+\Delta y_{i}\right)} \rho_{i}^{\mathrm{f}}(\mathbf{x}, \mathbf{y}) .
\end{aligned}
$$

Given the simple explicit dependence of the beam crosssection area and bending stiffness on the diameter,

$$
\begin{aligned}
A\left(y_{i}\right) & =\pi d\left(y_{i}-d\right), \\
E I_{i}\left(y_{i}\right) & =E \frac{\pi}{64}\left(y_{i}^{4}-\left(y_{i}-2 d\right)^{4}\right),
\end{aligned}
$$

where $d=2 \mathrm{~mm}$ is the wall thickness of the beam, it is straightforward to use (16) to approximate the solution to (14).

\section{2 | Geometric objective functions}

The objective functions (6a) and (8) require a large number of TZs to be considered. Even with the moderate number $n=22$ of modules considered here, spatial binning and evaluation of the angular distributions of the exit modules is a computationally costly operation, as it requires an exponentially growing number $O\left(2^{n}\right)$ of TZs to be generated and verified for self-intersections.

The considered projections of TZs onto the horizontal plane form a binary tree with each successive module represented by a trapezoid and being either $\mathrm{R}$ or $\mathrm{L}$. Therefore, for generation of all the TZs, a depth-first tree search is implemented with pruning at detected self-intersection. At each tree level, the last trapezoid is checked for intersections with all the preceding trapezoids besides the last five. That is, the $n$th trapezoid is checked with the trapezoids $1,2, \ldots, n-5$ (the four very last trapezoids are omitted, as self-intersection of such a short TZ is not possible). Given two trapezoids to be checked for intersection, the distance between their centers is first computed. Only if it is smaller than a threshold value, the full intersection check is triggered, which involves a pairwise verification of the trapezoid line segments: the exit and side segments of the current trapezoid are paired with the side segments of the preceding trapezoids (if it is the tree root, then its entrance segment is additionally considered). Finally, the intersection check for two line segments $\left(\mathbf{u}_{1}, \mathbf{u}_{2}\right)$ and $\left(\mathbf{v}_{1}, \mathbf{v}_{2}\right)$ is based on the determination of the clockwise/anticlockwise angular order of the following two pairs of point triples: $\left(\mathbf{u}_{1}, \mathbf{u}_{2}, \mathbf{v}_{1}\right)$ and $\left(\mathbf{u}_{1}, \mathbf{u}_{2}, \mathbf{v}_{2}\right)$, as well as $\left(\mathbf{v}_{1}, \mathbf{v}_{2}, \mathbf{u}_{1}\right)$ and $\left(\mathbf{v}_{1}, \mathbf{v}_{2}, \mathbf{u}_{2}\right)$. The line segments intersect if and only if

$$
\operatorname{det}\left[\begin{array}{c}
\mathbf{u}_{2}^{\mathrm{T}}-\mathbf{u}_{1}^{\mathrm{T}} \\
\mathbf{v}_{1}^{\mathrm{T}}-\mathbf{u}_{2}^{\mathrm{T}}
\end{array}\right] \operatorname{det}\left[\begin{array}{c}
\mathbf{u}_{2}^{\mathrm{T}}-\mathbf{u}_{1}^{\mathrm{T}} \\
\mathbf{v}_{2}^{\mathrm{T}}-\mathbf{p}_{2}^{\mathrm{T}}
\end{array}\right]<0
$$

and

$$
\operatorname{det}\left[\begin{array}{c}
\mathbf{v}_{2}^{\mathrm{T}}-\mathbf{v}_{1}^{\mathrm{T}} \\
\mathbf{u}_{1}^{\mathrm{T}}-\mathbf{v}_{2}^{\mathrm{T}}
\end{array}\right] \operatorname{det}\left[\begin{array}{c}
\mathbf{v}_{2}^{\mathrm{T}}-\mathbf{v}_{1}^{\mathrm{T}} \\
\mathbf{u}_{2}^{\mathrm{T}}-\mathbf{v}_{2}^{\mathrm{T}}
\end{array}\right]<0 .
$$

\subsection{Upper-level multicriterial optimization}

The multicriterial optimization problem stated at the beginning of this section constitutes the upper level of the considered approach. The optimization variables are the geometric parameters $\mathbf{x}$ that define the shape of the module. The lower-level procedures described above are repeatedly invoked to provide the geometric and structural objective functions. The upper-level optimization balances them by means of the Pareto front.

Determination of the Pareto front requires an application of a dedicated multiobjective optimization algorithm (A. Zhou et al. 2011). Here, the non-dominated sorting genetic algorithm II (NSGA-II) is used (Deb et al. 2002), which promotes a relatively uniform sampling of the front.

\section{6 | RESULTS}

The objective functions are sampled using a $2.5 \mathrm{~cm} \times 1^{\circ}$ domain sampling grid and plotted in Fig. 7 . The contradictory character of the geometrical and structural objectives and the need for multicriterial optimization are evident:

- The structural objective $m(\mathbf{x})$ promotes mid-length modules with a small planar angle. This is relatively intuitive, since such modules are able to generate more straight TZs that are less prone to twisting under load.

- Both geometric objectives $I_{\mathrm{xy}}(\mathbf{x})$ and $I_{\alpha}(\mathbf{x})$ promote long modules with the planar angle in the range between $32^{\circ}$ and $35^{\circ}$. Note that divisors of the full angle, such as $30^{\circ}$ and $36^{\circ}$, are discouraged, which is due to the increased overlapping and clustering of the $\mathrm{TZ}$ exit points. Small irregularities of the computed $I(\mathbf{x})$ are artifacts related to the specific choice of the bin grid.

The two proposed geometric objective functions, $I_{\mathrm{xy}}(\mathbf{x})$ and $I_{\alpha}(\mathbf{x})$, despite being defined in different ways, turn out to be strongly correlated, see Fig. 7 (top right). The high correlation of $92 \%$ confirms the consistent character of the proposed notion of geometric versatility.

Due to their high correlation, the two geometric objectives are balanced separately against the structural objective, which yields two separate (but similar) Pareto fronts. Figure 8 plots these fronts, as determined by means of NSGA-II, together 

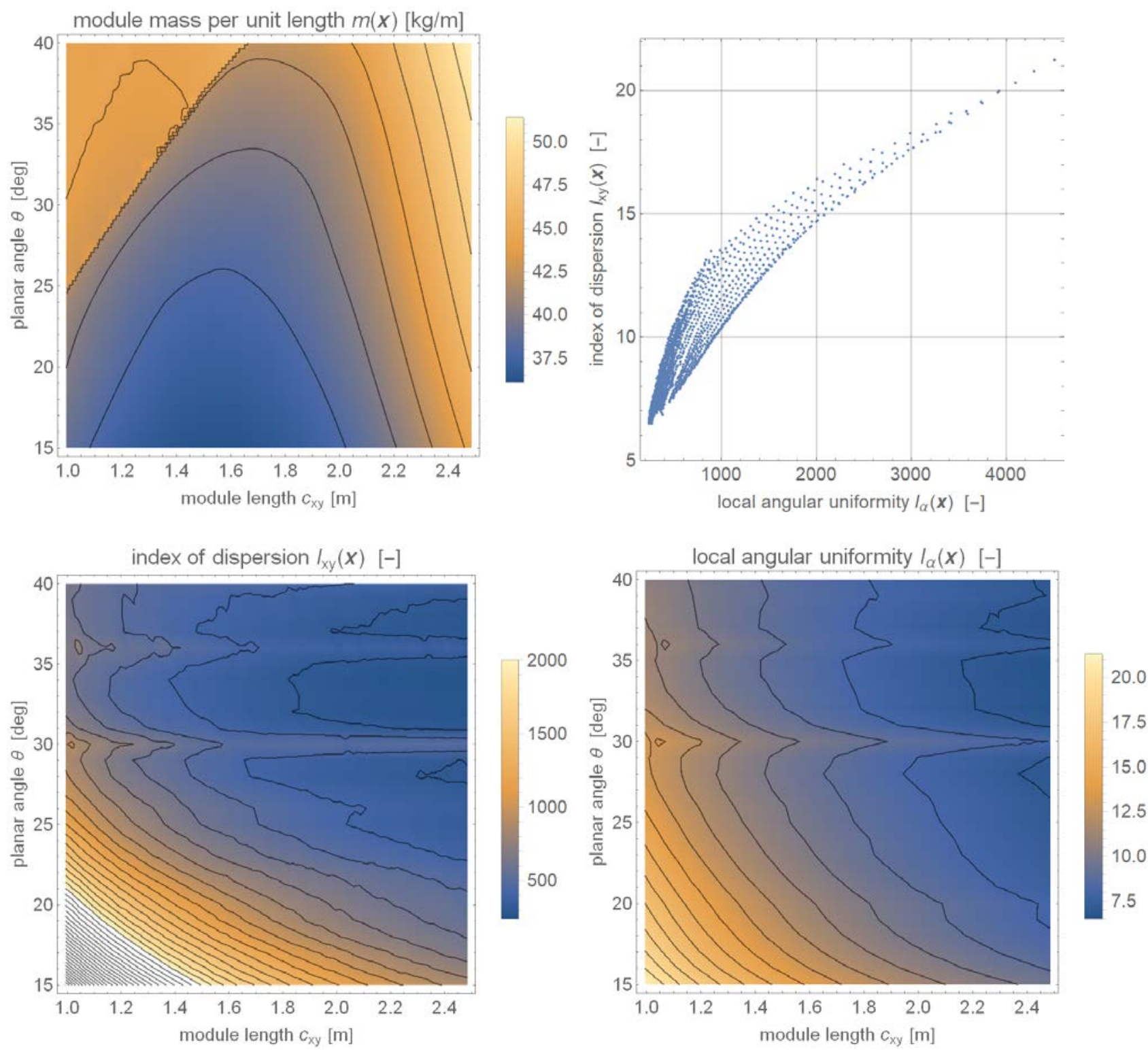

FIGURE 7 The considered objective functions: (top left) The structural objective function $m(\mathbf{x})$ : module mass per unit length (12); (top right) The scatter plot of the two geometric objective functions $I_{\mathrm{xy}}(\mathbf{x})$ and $I_{\alpha}(\mathbf{x})$; (bottom left) The geometric objective function $I_{\mathrm{xy}}(\mathbf{x})$ : index of dispersion 6a); (bottom right) The geometric objective function $I_{\alpha}(\mathbf{x})$ ) local angular uniformity 8 8).

with ten selected representative points and the image of the sampling grid $2.5 \mathrm{~cm} \times 1^{\circ}$. The corresponding Pareto sets in the search domain $\mathbf{x}=\left(\theta, c_{\mathrm{xy}}\right)$ are shown in Fig. 9 . Both Pareto sets are similar: the optimum module changes from the structurally optimum mid-length and possibly straight module, through balanced mid-length $32^{\circ}$-module, to the geometrically most versatile long $34^{\circ}$-module. The horizontal segments of the Pareto sets correspond to the horizontal valleys of the geometric objectives, see the bottom plots in Fig. 7 The relatively smooth variation of the beam diameters in the characteristic points along the identified Pareto fronts is shown in Fig. 10

\section{7 | CONCLUSION}

This paper proposes a multicriterial approach for optimization of free-form modular structures and illustrates it using an example of a skeletal, geometrically irregular modular ramp system for pedestrians. Two very different optimality measures have been considered. One is geometrical in nature and represents the ability of the module to create free-form ramps of diverse shapes that possibly uniformly fill the spatial environment. The other measure is structural in nature and defined as the minimum mass to length ratio of the module 

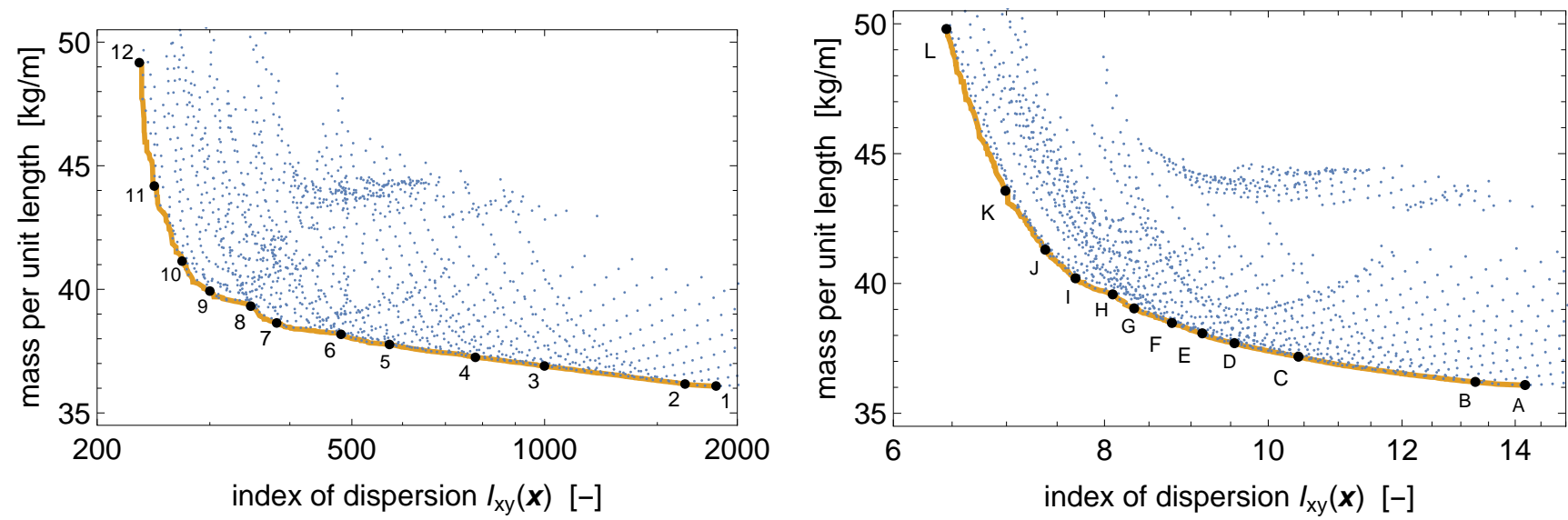

FIGURE 8 The point clouds in the domains $\left(I_{\mathrm{xy}}(\mathbf{x}), m(\mathbf{x})\right)$ (left) and $\left(I_{\alpha}(\mathbf{x}), m(\mathbf{x})\right)$ (right) of the objective functions that correspond to the $2.5 \mathrm{~cm} \times 1^{\circ}$ sampling grid of the search domain, shown together with the Pareto fronts (determined with NSGA-II) and twelve selected representative points.
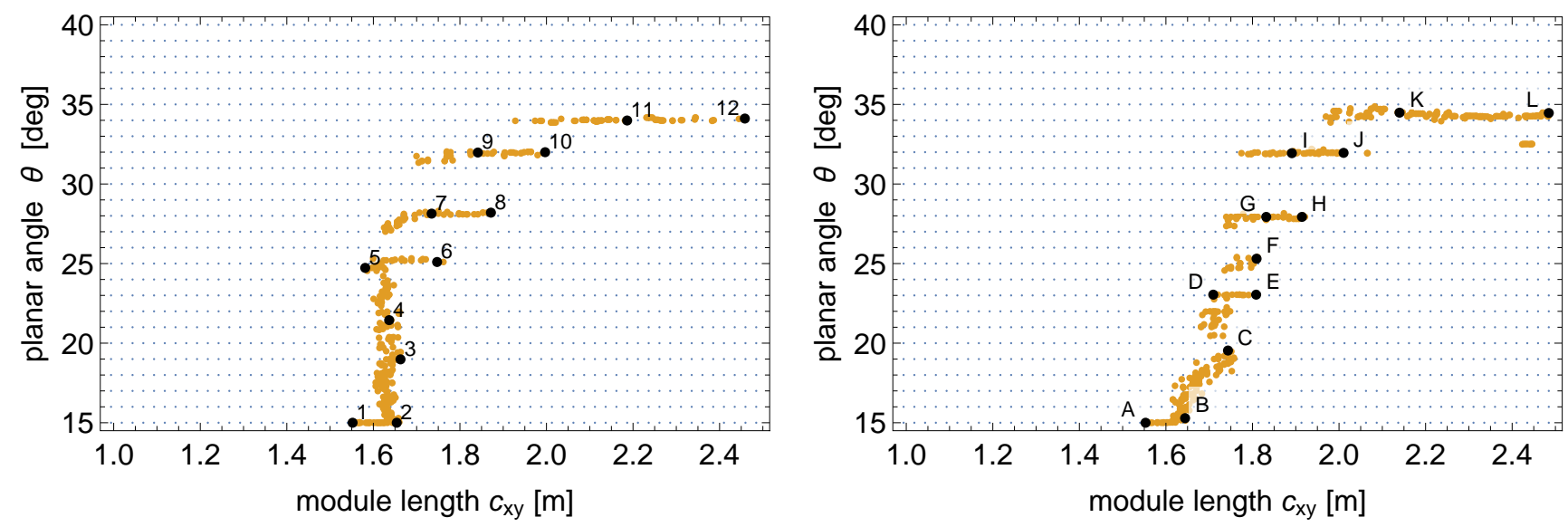

FIGURE 9 The Pareto sets in the search domain that correspond to the two determined Pareto fronts and the twelve representative points shown in Fig. 8

that allows any five-module ramp branch, subjected to a given design load, to satisfy upper bound constraints on the von Mises stress and local compressive forces. Both measures are computationally expensive: the former requires a large number of global structures to be generated and each verified for self-intersections, while the latter involves an inner optimization loop with respect to structural variables that needs to be performed for a large number of global configurations. The optimization variables represent local geometry of the module. The multiobjective evolutionary algorithm NSGA-II is used to find the Pareto front and balance the objectives.

The relatively high computational cost of the proposed objectives is related to the number of global configurations that grows exponentially with the number of available modules. It limits the proposed approach to structures composed of a moderate number of modules. Optimization of larger systems requires targeted techniques, such as determination of representative modular configurations, stochastic sampling of the configurations and GPU-based parallelization.

In a further perspective, the research is ongoing to consider additional criteria related to the construction costs, which has been shown in Sarma \& Adeli (2000) to result in substantial cost savings, as well as dynamic loading conditions $(\mathrm{Xu}$, Spencer, Lu, Chen, \& Lu, 2017), including crowd-structure interaction (Zawidzki, Chraibi, \& Nishinari, 2014), and to exploit the congruency of modules in a modular approach to local monitoring (Hou, Jankowski, \& Ou, 2013), decentralized structural control (Bakule, Rehák, \& Papík, 2016. El-Khoury \& Adeli 2013, Gutierrez Soto \& Adeli. 2017) and decentralized vibration damping (Pisarski, 2018, Poplawski, 


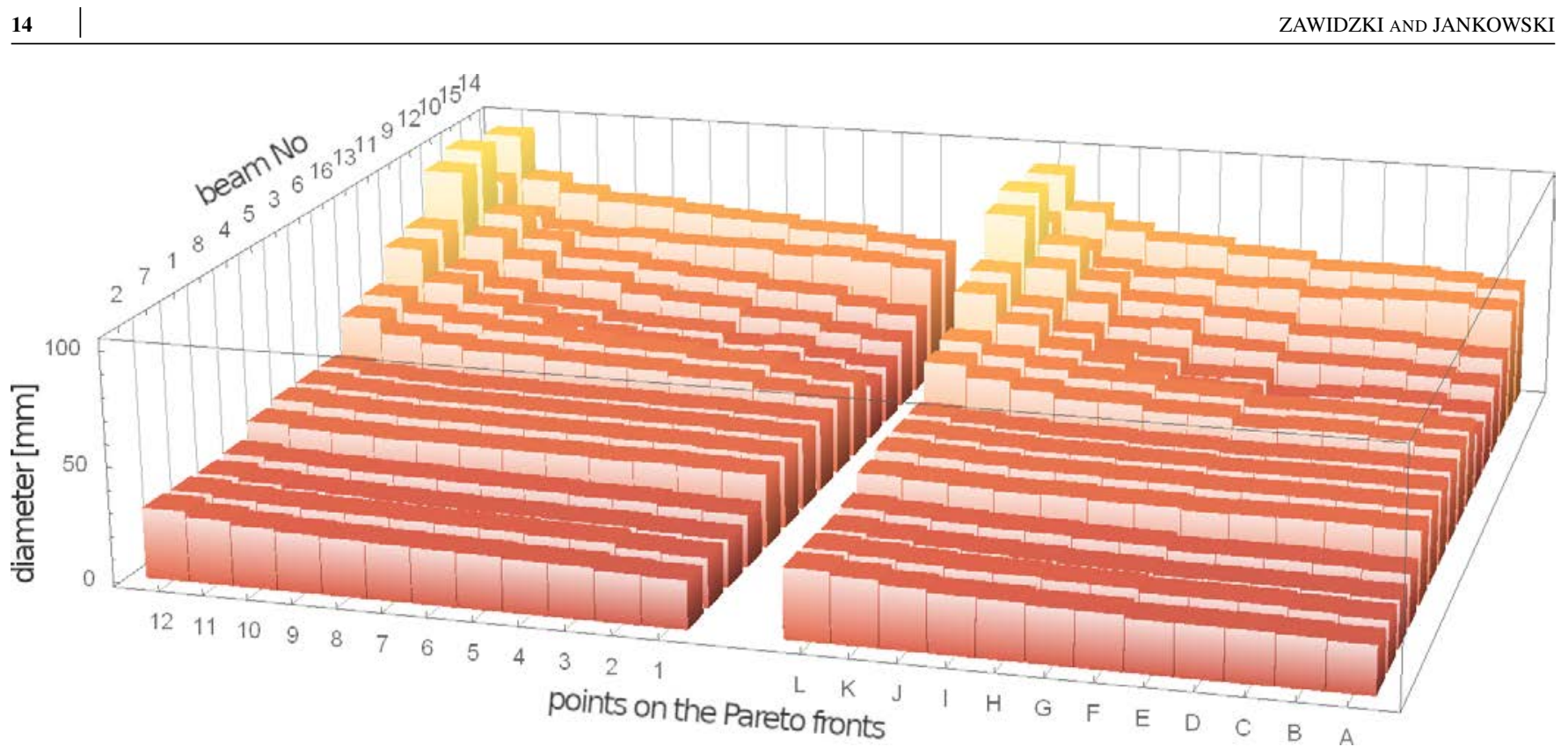

FIGURE 10 Beam diameters in the modules on the identified Pareto fronts. The beams are numbered as shown in Figure 1 while their sequence is rearranged to improve visibility.

Mikułowski, Mróz, \& Jankowski. 2018). An approach similar to Kociecki \& Adeli (2014) can be exploited to include simultaneous optimization of the internal topology of the module.

\section{ACKNOWLEDGMENTS}

This research is a part of the project titled "Innovative Extremely Modular Systems for temporary and permanent deployable structures and habitats: development, modeling, evaluation \& optimization" and funded by "Polonez 2" research grant No 2016/21/P/ST8/03856 supported by the National Science Centre, Poland. This project has received funding from the European Union's Horizon 2020 research and innovation programme under the Marie Skłodowska-Curie grant agreement No 665778,

\section{References}

ADA Standards for Accessible Design. (2010). Washington, D.C.: Dept. of Justice.

Adeli, H., \& Balasubramanyam, K. V. (1987). Interactive layout optimization of trusses. Journal of Computing in Civil Engineering, 1(3), 183-196. doi:

Adeli, H., \& Kamal, O. (1986). Efficient optimization of space trusses. Computers \& Structures, 24(3), 501-511. doi:

Adeli, H., \& Kamal, O. (1991). Efficient optimization of plane trusses. Advances in Engineering Software and
Workstations, 13(3), 116-122. doi:

Augustyniak, M. (2018). Old materials - new capabilities: lattice materials in structural mechanics. Journal of Theoretical and Applied Mechanics, 56(1), 213-226. doi:

Bakule, L., Rehák, B., \& Papík, M. (2016). Decentralized networked control of building structures. Computer-Aided Civil and Infrastructure Engineering, 31(11), 871-886. doi:

Chen, N., Yu, D., Xia, B., Liu, J., \& Ma, Z. (2017). Microstructural topology optimization of structural-acoustic coupled systems for minimizing sound pressure level. Structural and Multidisciplinary Optimization, 56(6), 1259-1270. doi:

Chen, Y., Zhou, S., \& Li, Q. (2010). Multiobjective topology optimization for finite periodic structures. Computers \& Structures, 88(11), 806 - 811. doi:

Cressie, N. (2015). Statistics for spatial data, revised edition. John Wiley \& Sons. doi:

Deaton, J. D., \& Grandhi, R. V. (2013). A survey of structural and multidisciplinary continuum topology optimization: post 2000. Structural and Multidisciplinary Optimization, 49(1), 1-38. doi:

Deb, K., Pratap, A., Agarwal, S., \& Meyarivan, T. (2002). A fast and elitist multiobjective genetic algorithm: NSGAII. IEEE Transactions on Evolutionary Computation, 6(2), 182-197. doi:

Ehrgott, M. (2005). Multicriteria optimization. SpringerVerlag. doi: 
El-Khoury, O., \& Adeli, H. (2013). Recent advances on vibration control of structures under dynamic loading. Archives of Computational Methods in Engineering, 20(4), 353-360. doi:

Feliciani, C., \& Nishinari, K. (2016). An improved cellular automata model to simulate the behavior of high density crowd and validation by experimental data. Physica A: Statistical Mechanics and its Applications, 451, 135-148. doi:

Gilbert, M., \& Tyas, A. (2003). Layout optimization of large-scale pin-jointed frames. Engineering Computations, 20(8), 1044-1064. doi:

Gutierrez Soto, M., \& Adeli, H. (2017). Many-objective control optimization of high-rise building structures using replicator dynamics and neural dynamics model. Structural and Multidisciplinary Optimization, 56(6), 15211537. doi:

Hou, J., Jankowski, Ł., \& Ou, J. (2013). An online substructure identification method for local structural health monitoring. Smart Materials and Structures, 22(9), 095017. doi:

Illian, J., Penttinen, A., Stoyan, H., \& Stoyan, D. (2008). Statistical analysis and modelling of spatial point patterns. John Wiley \& Sons. doi:

Kociecki, M., \& Adeli, H. (2014). Two-phase genetic algorithm for topology optimization of free-form steel space-frame roof structures with complex curvatures. Engineering Applications of Artificial Intelligence, 32, 218-227. doi:

Kociecki, M., \& Adeli, H. (2015). Shape optimization of free-form steel space-frame roof structures with complex geometries using evolutionary computing. Engineering Applications of Artificial Intelligence, 38, 168-182. doi:

Li. W., Pu, H., Schonfeld, P., Song, Z., Zhang, H., Wang, L., Wang, J., Peng, X., \& Peng, L. (2018). A method for automatically recreating the horizontal alignment geometry of existing railways. Computer-Aided Civil and Infrastructure Engineering, 34(1), 71-94. doi:

Liu, L., Yan, J., \& Cheng, G. (2008). Optimum structure with homogeneous optimum truss-like material. Computers \& Structures, 86(13-14), 1417-1425. doi:

LRFD Guide Specification for the Design of Pedestrian Bridges. (2009). American Association of State Highway and Transporation Officials (AASHTO).

Marler, T. R., \& Arora, J. S. (2004). Survey of multiobjective optimization methods for engineering. Structural and Multidisciplinary Optimization, 26(6), 369-395. doi:

Messner, M. C. (2016). Optimal lattice-structured materials. Journal of the Mechanics and Physics of Solids, 96, 162183. doi:
Moses, E., Fuchs, M. B., \& Ryvkin, M. B. (2002). Topological design of modular structures under arbitrary loading. Structural and Multidisciplinary Optimization, 24(6), 407-417. doi:

Niu, B., Yan, J., \& Cheng, G. (2008). Optimum structure with homogeneous optimum cellular material for maximum fundamental frequency. Structural and Multidisciplinary Optimization, 39(2), 115-132. doi:

Patnaik, S. N., \& Hopkins, D. A. (1998). Optimality of a fully stressed design. Computer Methods in Applied Mechanics and Engineering, 165(1-4), 215-221. doi:

Pisarski, D. (2018). Decentralized stabilization of semiactive vibrating structures. Mechanical Systems and Signal Processing, 100, 694-705. doi:

Plevris, V., \& Papadrakakis, M. (2010). A hybrid particle swarm-gradient algorithm for global structural optimization. Computer-Aided Civil and Infrastructure Engineering. doi:

Poplawski, B., Mikułowski, G., Mróz, A., \& Jankowski, Ł. (2018). Decentralized semi-active damping of free structural vibrations by means of structural nodes with an on/off ability to transmit moments. Mechanical Systems and Signal Processing, 100, 926-939. doi:

Sarma, K. C., \& Adeli, H. (2000). Fuzzy discrete multicriteria cost optimization of steel structures. Journal of Structural Engineering, 126(11), 1339-1347. doi:

Sigmund, O., \& Maute, K. (2013). Topology optimization approaches. Structural and Multidisciplinary Optimization, 48(6), 1031-1055. doi:

Smith, R. E. (2011). Prefab architecture: a guide to modular design and construction. John Wiley \& Sons.

Sokół, T. (2011). A 99 line code for discretized Michell truss optimization written in Mathematica. Structural and Multidisciplinary Optimization, 43(2), 181-190. doi:

Torstenfelt, B. R., \& Klarbring, A. (2006, 01). Structural optimization of modular product families with application to car space frame structures. Structural and Multidisciplinary Optimization, 32(2), 133-140. doi:

Tugilimana, A., Thrall, A. P., \& Coelho, R. F. (2017). Conceptual design of modular bridges including layout optimization and component reusability. Journal of Bridge Engineering, 22(11), 04017094. doi:

Tugilimana, A., Thrall, A. P., Descamps, B., \& Coelho, R. F. (2017). Spatial orientation and topology optimization of modular trusses. Structural and Multidisciplinary Optimization, 55(2), 459-476. doi:

Wang, Y., Luo, Z., Zhang, N., \& Qin, Q. (2016). Topological shape optimization of multifunctional tissue engineering scaffolds with level set method. Structural and Multidisciplinary Optimization, 54(2), 333-347. doi: 
Weidmann, U. (1993). Transporttechnik der fussgänger: Transporttechnische eigenschaften des fussgängerverkehrs, literaturauswertung (Vol. 90). ETH Zurich. doi:

Xinchao, Z. (2011). Simulated annealing algorithm with adaptive neighborhood. Applied Soft Computing, 11(2), 1827-1836. doi:

Xu, J., Spencer, B. F., Lu, X., Chen, X., \& Lu, L. (2017). Optimization of structures subject to stochastic dynamic loading. Computer-Aided Civil and Infrastructure Engineering, 32(8), 657-673. doi:

Zawidzki, M. (2015). Retrofitting of pedestrian overpass by Truss-Z modular systems using graph-theory approach. Advances in Engineering Software, 81, 41-49. doi:

Zawidzki, M. (2016). Optimization of multi-branch Truss$\mathrm{Z}$ based on evolution strategy. Advances in Engineering Software, 100, 113-125. doi:

Zawidzki, M., Chraibi, M., \& Nishinari, K. (2014). Crowd-Z: The user-friendly framework for crowd simulation on an architectural floor plan. Pattern Recognition Letters, 44, 88-97. doi:

Zawidzki, M., \& Jankowski, Ł. (2017). Multicriterial optimization of geometrical and structural properties of the basic module of a single-branch Truss- $Z$ structure. In Advances in structural and multidisciplinary optimization (pp. 163-174). Springer International Publishing. doi:
Zawidzki, M., \& Jankowski, Ł. (2018). Optimization of modular Truss- $Z$ by minimum-mass design under equivalent stress constraint. Smart Structures and Systems, 21(6), 715-725. doi:

Zawidzki, M., \& Szklarski, J. (2018). Effective multiobjective discrete optimization of truss-z layouts using a GPU. Applied Soft Computing, 70, 501-512. doi:

Zegard, T., \& Paulino, G. H. (2015). GRAND3 - Ground structure based topology optimization for arbitrary 3D domains using MATLAB. Structural and Multidisciplinary Optimization, 52(6), 1161-1184. doi:

Zhou, A., Qu, B.-Y., Li, H., Zhao, S.-Z., Suganthan, P. N., \& Zhang, Q. (2011). Multiobjective evolutionary algorithms: A survey of the state of the art. Swarm and Evolutionary Computation, 1(1), 32-49. doi:

Zhou, C. W., Lainé, J. P., Ichchou, M., \& Zine, A. M. (2015). Multi-scale modelling for two-dimensional periodic structures using a combined mode/wave based approach. Computers \& Structures, 154, 145 - 162. doi:

How to cite this article: Zawidzki M., Jankowski Ł., Multiobjective optimization of modular structures: weight versus geometric versatility in a Truss-Z system. Comput Aided Civ Inf., 2019;00:0-0. 